\title{
A Novel GPS Antijamming Receiver Based on Noncircularity
}

\author{
Chen Xian-Ning, Qun Wan, and Yang Wan-Lin \\ Department of Electronic Engineering, University of Electronic Science and Technology of China, Sichuan Chengdu 610054, China
}

Correspondence should be addressed to Chen Xian-Ning, xianning.chen@gmail.com

Received 25 May 2009; Accepted 2 October 2009

Academic Editor: Muhammad Taher Abuelma'atti

Copyright ( $\odot 2010$ Chen Xian-Ning et al. This is an open access article distributed under the Creative Commons Attribution License, which permits unrestricted use, distribution, and reproduction in any medium, provided the original work is properly cited.

\begin{abstract}
We present a novel antijamming global position system (GPS) receiver that relies on the unique noncircularity of the GPS signal. Since the GPS signal is BPSK modulated, it is a noncircular signal while most interferers are circular signals. The proposed receiver utilizes this circular difference property to excise interferences while preserve the GPS signals. It is shown that the proposed receiver is a blind receiver that does not require any angular information of the satellites.
\end{abstract}

\section{Introduction}

A Global Positioning System (GPS) is a satellite-based allweather navigation system and having a large success in civil application because its low prize receives its global coverage and its precision in navigation solutions [1]. However, the intentional interferences and the increasing pollution of electromagnetic environment may make GPS receivers lose their efficacy. Interference suppression in GPS can be conducted in the time, space, or frequency domain, or in a domain of joint variables [2-4]. It is noted, however, that the existing GPS interference cancellation techniques do not fully utilize the noncircularity property of the GPS signal.

Recently, more and more researches concerned on using the non-circularity of signals to improve the performance of signal processing such as root-MUSIC algorithm [5] and enhanced unitary ESPRIT [6] for non-circular sources DOA estimation, enhanced blind estimator [7], and nondata aided carrier frequency offset estimation in non-circular transmissions through frequency-selective channels [8]. GPS signal is BPSK modulated and is a non-circular signal while most interferers are circular signals.

This letter proposes a new interference suppression technique using, in effect, the circular difference between the GPS signal and the interferes. Unlike previous contributions in this area, the proposed GPS receiver exploits the unique noncircular property to suppress a large class of narrowband and broadband interferers. Furthermore, neither the knowledge of the satellite locations nor the synchronization between the satellite and the receiver is required to perform interference cancellation.

Section 2 describes the system model and introduces the proposed method. Section 3 will present simulation results and show that the proposed method can achieve a similar performance as an MMSE receiver. Section 4 will make conclusions.

\section{Proposed Antijamming Receiver}

A block diagram of the GPS receiver with an M-element spatial uniform linear array(ULA) is depicted in Figure 1.

The waveforms impinging on the array are those of the GPS signals, interferences, and noise. After down-conversion and sampled by chip-rate, the discrete-time format of the received signal vector form can be presented as

$$
\mathbf{x}(n)=\mathbf{a}_{s} \cdot s(n)+\sum_{p=1}^{P} \mathbf{a}_{j p} \cdot j_{p}(n)+\mathbf{v}(n),
$$

where $s(n)$ is signal component, as steer vector of the signal; $\mathbf{a}_{s}=\left[1, e^{j 2 \pi(d / c) \sin \theta_{s}}, \ldots, e^{j 2 \pi(d / c)(M-1) \sin \theta_{s}}\right]^{T} \theta_{s}$ is steer vector of the GPS signal, where $d$ is the sensor spacing, $c$ is the propagation speed of the waveform, and $\theta_{s}$ represents the arriving angle of the signal; $j_{p}(n)$ is the waveform of the $p$ th interfere; $\mathbf{a}_{j p}=\left[1, e^{j 2 \pi(d / c) \sin \theta_{j p}}, \ldots, e^{j 2 \pi(d / c)(M-1) \sin \theta_{j p}}\right]^{T}$ is steer vector of the $p$ th interferer, where $\theta_{j p}$ represents the 


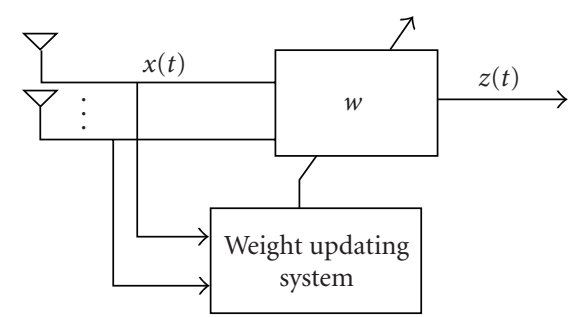

FIgURE 1: Block diagram of the GPS receiver.

arriving angle of the $p$ th interferer; $\mathbf{v}(n)$ is additive white Gaussian noise sample vector with variance $\sigma_{v}$.

Equation (1) can be rewritten as

$$
\mathbf{x}(n)=\mathbf{s}(n)+\mathbf{j}(n)+\mathbf{v}(n)
$$

where $\mathbf{s}(n)$ denote the signal vector and $\mathbf{j}(n)=\sum_{p=1}^{P} \mathbf{a}_{j p}$. $j_{p}(n)$ is the compound interference vector.

Under the assumption that GPS signals, interference, and noise are independent, the normal covariance matrix $\mathbf{R}_{x x}$ of the received signal becomes

$$
\mathbf{R}_{x x}=E\left\{\mathbf{x}(n) \cdot \mathbf{x}^{H}(n)\right\}=\mathbf{R}_{s}+\mathbf{R}_{j}+\mathbf{R}_{v},
$$

where $E\{\cdot\}$ represents the statistical expectation. $(\cdot)^{H}$ denotes complex conjugate transpose, and $\mathbf{R}_{s}, \mathbf{R}_{j}$, and $\mathbf{R}_{v}$ are covariance matrices of the GPS signals, the interference, and the noise, which are defined, respectively, as

$$
\begin{gathered}
\mathbf{R}_{s} \triangleq E\left\{\mathbf{s}(n) \cdot \mathbf{s}^{H}(n)\right\}, \\
\mathbf{R}_{j} \triangleq E\left\{\mathbf{j}(n) \cdot \mathbf{j}^{H}(n)\right\}, \\
\mathbf{R}_{v} \triangleq E\left\{\mathbf{v}(n) \cdot \mathbf{v}^{H}(n)\right\}=\sigma_{v} \mathbf{I},
\end{gathered}
$$

where $\mathbf{I}$ is an $M \times M$ identity matrix.

GPS signal is BPSK modulated and is a non-circular signal while jammers and noise are circular signals. The elliptic covariance matrix $\mathbf{R}_{x x}^{\prime}$ of the received signal should be

$$
\mathbf{R}_{x x}^{\prime}=E\left\{\mathbf{x}(n) \cdot \mathbf{x}^{T}(n)\right\}=\mathbf{R}_{s}^{\prime}+\mathbf{R}_{j}^{\prime}+\mathbf{R}_{v}^{\prime},
$$

where $(\cdot)^{T}$ denotes transpose, and $\mathbf{R}_{s}^{\prime}, \mathbf{R}_{j}^{\prime}$, and $\mathbf{R}_{v}^{\prime}$ are elliptic covariance matrices of the GPS signals, the interference, and the noise. To circular signals, its elliptic covariance matrix is equal to zero, which means that

$$
\begin{array}{r}
\mathbf{R}_{j}^{\prime} \triangleq E\left\{\mathbf{j}(n) \cdot \mathbf{j}^{T}(n)\right\}=0, \\
\mathbf{R}_{v}^{\prime} \triangleq E\left\{\mathbf{v}(n) \cdot \mathbf{v}^{T}(n)\right\}=0 .
\end{array}
$$

Then, (5) becomes

$$
\mathbf{R}_{x x}^{\prime}=\mathbf{R}_{s}^{\prime}
$$

Let $\mathbf{w}$ be the $M \times 1$ weight vector. Then, the output of the beamformer is given by

$$
\mathbf{z}(n)=\mathbf{w}^{H} \cdot \mathbf{x}(n),
$$

and the beamformer $\mathbf{w}$ is obtained by Maximizing the Noncircular to Circular Ratio (MNCR) of the output signal:

$$
\begin{aligned}
\mathbf{w}_{\text {MNCR }} & =\underset{\mathbf{w}}{\operatorname{argmax}} \frac{E\left\{\left|\mathbf{z}(n) \cdot \mathbf{z}^{T}(n)\right|\right\}}{E\left\{\left|\mathbf{z}(n) \cdot \mathbf{z}^{H}(n)\right|\right\}} \\
& =\underset{\mathbf{w}}{\operatorname{argmax}} \frac{\left|\mathbf{w}^{H} \mathbf{R}_{x x}^{\prime} \mathbf{w}^{*}\right|}{\mathbf{w}^{H} \mathbf{R}_{x x} \mathbf{w}},
\end{aligned}
$$

where $(\cdot)^{*}$ denotes complex conjugate.

Since the denominator of (9) is positive real, we notice that (9) is unchanged when $\mathbf{w}$ undergoes an arbitrary phase rotation. Then, by choosing a $\mathbf{w}$ such that $\operatorname{Im}\left\{\mathbf{w}^{H} \mathbf{R}_{x x}^{(T)} \mathbf{w}^{*}\right\}=$ 0 where $\operatorname{Im}\{\cdot\}$ denotes the imagine part, then, (9) can be rewritten as

$$
\mathbf{w}_{\text {MNCR }}=\underset{\mathbf{w}}{\operatorname{argmax}} \frac{\operatorname{Re}\left\{\mathbf{w}^{H} \mathbf{R}_{x x}^{\prime} \mathbf{w}^{*}\right\}}{\operatorname{Re}\left\{\mathbf{w}^{H} \mathbf{R}_{x x} \mathbf{w}\right\}} .
$$

Let

$$
\begin{aligned}
\mathbf{w}_{r}=\operatorname{Re}\{\mathbf{w}\} ; & \mathbf{w}_{i}=\operatorname{Im}\{\mathbf{w}\}, \\
\mathbf{R}_{x x r}=\operatorname{Re}\left\{\mathbf{R}_{x x}\right\} ; & \mathbf{R}_{x x i}=\operatorname{Im}\left\{\mathbf{R}_{x x}\right\}, \\
\mathbf{R}_{x x r}^{\prime}=\operatorname{Re}\left\{\mathbf{R}_{x x}^{\prime}\right\} ; & \mathbf{R}_{x x i}^{\prime}=\operatorname{Im}\left\{\mathbf{R}_{x x}^{\prime}\right\},
\end{aligned}
$$

where $\operatorname{Re}\{\cdot\}$ denotes the real part. Then we can expand (10) in complex form as

$\mathbf{w}_{\text {MNCR }}$

$$
\begin{aligned}
& =\underset{\left(\mathbf{w}_{r}, \mathbf{w}_{i}\right)}{\operatorname{argmax}} \frac{\operatorname{Re}\left\{\left(\mathbf{w}_{r}^{T}-j \mathbf{w}_{i}^{T}\right)\left(\mathbf{R}_{x x r}^{\prime}+j \mathbf{R}_{x x i}^{\prime}\right)\left(\mathbf{w}_{r}-j \mathbf{w}_{i}\right)\right\}}{\left.\operatorname{Re}\left\{\mathbf{w}_{r}^{T}-j \mathbf{w}_{i}^{T}\right)\left(\mathbf{R}_{x x r}+j \mathbf{R}_{x x i}\right)\left(\mathbf{w}_{r}+j \mathbf{w}_{i}\right)\right\}} \\
& =\underset{\overline{\mathbf{w}}}{\operatorname{argmax}} \frac{\overline{\mathbf{w}}^{T}\left(\overline{\mathbf{R}}_{x x}^{\prime}\right) \overline{\mathbf{w}}}{\overline{\mathbf{w}}^{T}\left(\overline{\mathbf{R}}_{x x}\right) \overline{\mathbf{w}}},
\end{aligned}
$$

where $j=\sqrt{-1}$ and $\overline{\mathbf{w}}=\left[\begin{array}{l}\mathbf{w}_{r} \\ \mathbf{w}_{i}\end{array}\right]$ is a $2 M \times 1$ real vector; $\overline{\mathbf{R}}_{x x}^{\prime}=$ $\left[\begin{array}{cc}\mathbf{R}_{x x r}^{\prime} & \mathbf{R}_{x x i}^{\prime} \\ \mathbf{R}_{x x i}^{\prime} & -\mathbf{R}_{x x r}^{\prime}\end{array}\right]$ and $\overline{\mathbf{R}}_{x x}=\left[\begin{array}{cc}\mathbf{R}_{x x r} & -\mathbf{R}_{x x i} \\ \mathbf{R}_{x x i} & \mathbf{R}_{x x r}\end{array}\right]$ are both $2 M \times 2 M$ real matrices.

Thus, the optimum $\overline{\mathbf{w}}$ is the eigenvector corresponding to the maximum eigenvalue of the following generalized eigenvalue problem:

$$
\overline{\mathbf{R}}_{x x}^{\prime} \cdot \overline{\mathbf{w}}=\lambda_{\max } \cdot \overline{\mathbf{R}}_{x x} \cdot \overline{\mathbf{w}},
$$

where $\lambda_{\max }$ denotes the maximum eigenvalue, which is also the maximum NCR.

In practice, $\mathbf{R}_{x x}^{\prime}$ and $\mathbf{R}_{x x}$ are replaced by their sample estimate:

$$
\begin{aligned}
& \hat{\mathbf{R}}_{x x}^{\prime} \triangleq \frac{1}{K} \mathbf{X}_{K} \cdot \mathbf{X}_{K}^{T}, \\
& \hat{\mathbf{R}}_{x x} \triangleq \frac{1}{K} \mathbf{X}_{K} \cdot \mathbf{X}_{K}^{H},
\end{aligned}
$$

where $M \times K$ data matrix is $\mathbf{X}_{K} \triangleq[\mathbf{x}(n), \ldots, \mathbf{x}(n-(K-1)]$. 


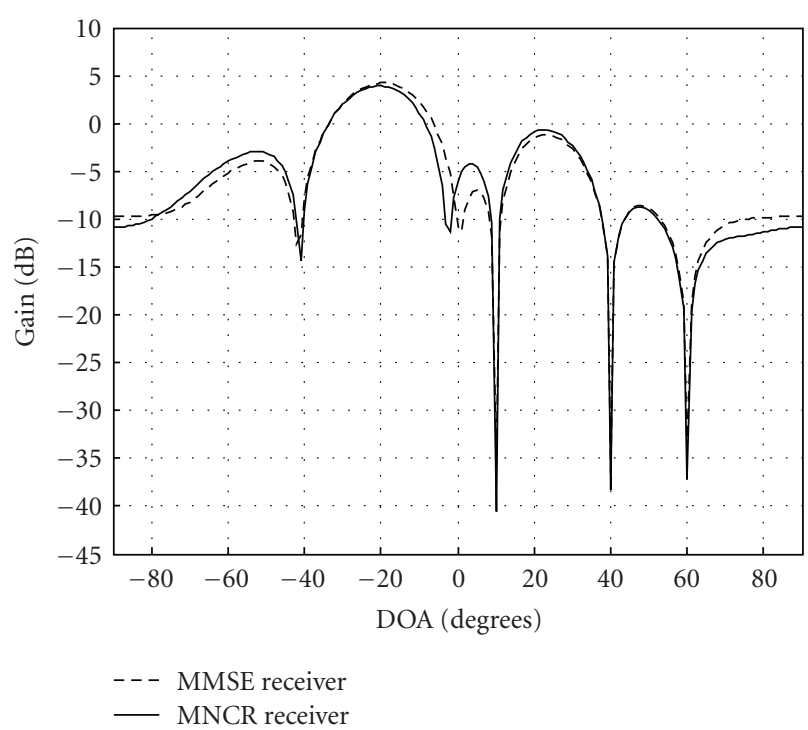

FIGURE 2: Comparison of Antenna gains between the self-coherence receiver and the MMSE receiver with $S N R=-20 \mathrm{~dB}$, JSR $=60 \mathrm{~dB}$.

The updating algorithm may now be outlined as follows:

(1) estimating $\hat{\mathbf{R}}_{x x}^{\prime}$ and $\hat{\mathbf{R}}_{x x}$ using data sample matrix $\mathbf{X}_{K}$;

(2) forming matrices $\overline{\mathbf{R}}_{x x}^{\prime}$ and $\overline{\mathbf{R}}_{x x}$ using real and imagine part of $\hat{\mathbf{R}}_{x x}^{\prime}$ and $\hat{\mathbf{R}}_{x x}$;

(3) determining an estimate $\overline{\mathbf{W}}$ according to (13);

(4) finally, the estimate of $\mathbf{w}_{\mathrm{MNCR}}$ can be written as

$$
\mathbf{w}_{\mathrm{MNCR}}=\mathbf{w}_{r}+j \mathbf{w}_{i}
$$

\section{Simulations}

A 7-element uniform linear array with half-wavelength spacing is used in the simulation. GPS navigation symbols are in the BPSK format and spread by the Gold code with processing gain $P=1023$. At the receiver, chip-rat sampling is performed, and $K=800$ samples are collected. Jammers used in the simulations are generated as broadband binary signals having the same rates as the $\mathrm{C} / \mathrm{A}$-codes but with a different structure than that of the C/A signals.

In the first simulation, to examine the performance of the proposed GPS receiver in presence of multiple strong jammers, we compare the proposed GPS receiver with the MMSE receiver in [4]. In the simulation, the satellite is located at $-20^{\circ}$, and the three jammers are at $10^{\circ}, 40^{\circ}$, and $60^{\circ}$. It is noted that the proposed receiver need no prior information of the satellite location while the MMSE receiver does need the knowledge in order to compute the power of the GPS signal. Figure 2 shows that both receivers are capable of suppressing strong jammer while preserving the GPS signal, with the proposed receiver having the advantage of being a blind receiver.

In the second simulation, consider the same condition of the first simulation; we examine the effectiveness of the

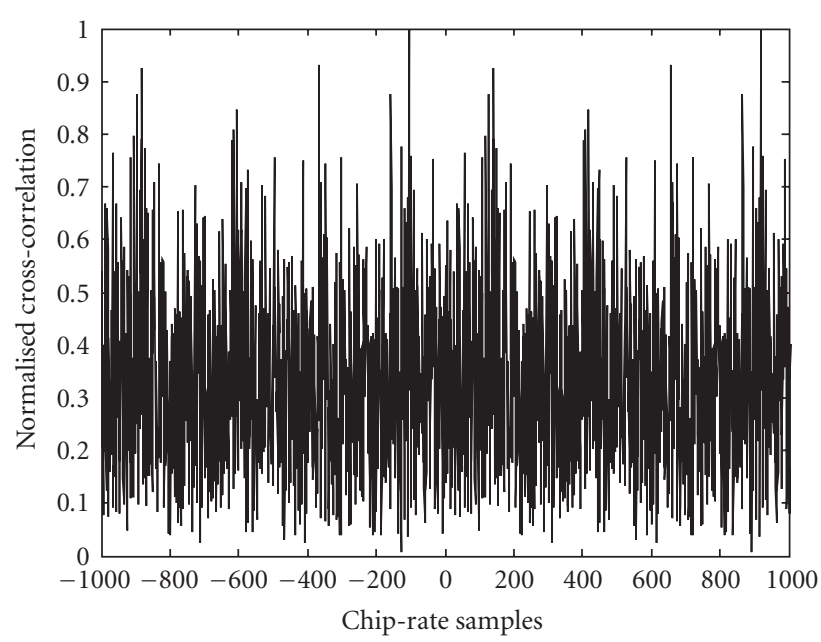

(a)

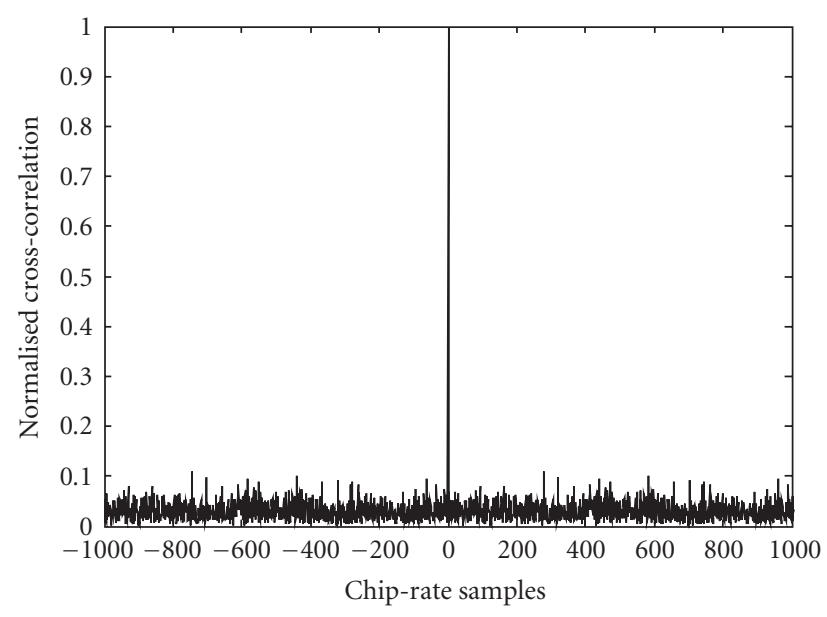

(b)

FIGURE 3: (a) Normalized cross-correlation before jammer removal. (b) Normalized cross-correlation after jammer removal.

proposed GPS receiver. In GPS, the receiver is ultimately evaluated based on its ability to provide accurate pseudorange measurements. This is achieved by establishing synchronization between the receiver and the satellite, which is decided based on the cross-correlation between the beamformer outputs and a locally generated Gold sequence [1]. The normalized cross-correlations before and after the jammer removal in Figure 3, show that the proposed GPS receiver can effectively cancel directional jammers and achieve synchronization even when the JSR is as high as $60 \mathrm{~dB}$ (Figure 3(b)). Without interference suppression, however, synchronization fails, as is evident in Figure 3(a).

\section{Conclusion}

We have presented a new GPS receiver that is based on the non-circularity of the GPS signals. Due to the different circularity between the GPS signal and interferers, we present 
new adaptive beamforming criteria-the Maximum Noncircular to Circular Ratio (MNCR) criteria. Utilizing this criterion, an anti-jamming GPS receiver is constructed to mitigate a wide class of interferers. Simulations have shown that the proposed receiver is capable of suppressing strong jammers while preserving GPS signals, without requiring any knowledge of the angular information of the satellites.

\section{Acknowledgments}

This work was supported in part by the National Natural Science Foundation of China under Grant 60772146, the National High Technology Research and Development Program of China (863 Program) under Grant 2008AA12Z306, and in part by Science Foundation of Ministry of Education of China under Grant 109139.

\section{References}

[1] E. D. Kaplan, Ed., Understanding GPS: Principles and Applications, Artech House, Norwell, Mass, USA, 1996.

[2] Y. Zhang, M. G. Amin, and A. R. Lindsey, "Anti-jamming GPS receivers based on bilinear signal distributions," in Proceedings of the IEEE Military Communications Conference (MILCOM '01), vol. 2, pp. 1070-1074, McLean, Va, USA, 2001.

[3] M. D. Zoltowski and A. S. Gecan, "Advanced adaptive null steer in concepts for GPS," in Proceedings of the IEEE Military Communications Conference (MILCOM '95), vol. 3, pp. 12141218, San Diego, Calif, USA, May 1995.

[4] R. L. Fante and J. J. Vaccaro, "Wideband cancellation of interference in a GPS receive array," IEEE Transactions on Aerospace and Electronic Systems, vol. 36, no. 2, pp. 549-564, 2000.

[5] P. Charge, Y. Wang, and J. Saillard, "A root-MUSIC algorithm for non circular sources," in Proceedings of the IEEE International Conference on Acoustics, Speech and Signal Processing (ICASSP '01), vol. 5, pp. 2985-2988, Salt Lake City, Utah, USA, May 2001.

[6] M. Haardt and F. Romer, "Enhancements of unitary ESPRIT for non-circular sources," in Proceedings IEEE International Conference on Acoustics, Speech and Signal Processing (ICASSP '04), vol. 2, pp. 101-104, Montreal, Canada, May 2004.

[7] P. Ciblat, E. Serpedin, and Y. Wang, "A fractionally-sampling based frequency offset enhanced blind estimator for noncircular transmissions," in Proceedings of the Conference Record of the 36th Asilomar Conference on Signals, Systems and Computers, vol. 1, pp. 659-663, November 2002.

[8] P. Ciblat, P. Loubaton, E. Serpedin, and G. B. Giannakis, "Performance of non-data aided carrier offset estimation for noncircular transmissions through frequency-selective channels," in Proceedings of the IEEE International Conference on Acoustics, Speech, and Signal Processing (ICASSP '00), pp. 2525-2528, Istanbul, Turkey, June 2000. 

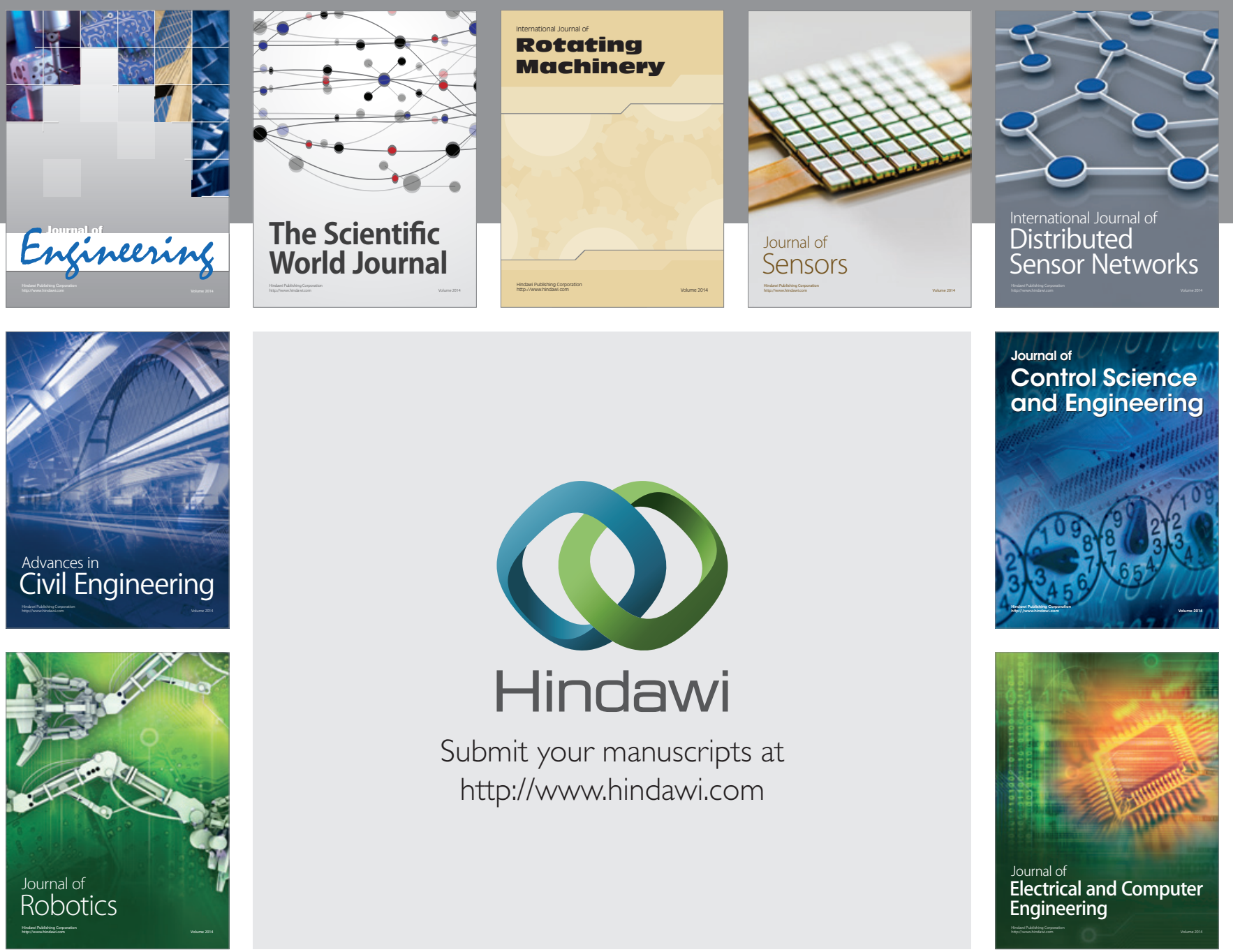

Submit your manuscripts at

http://www.hindawi.com
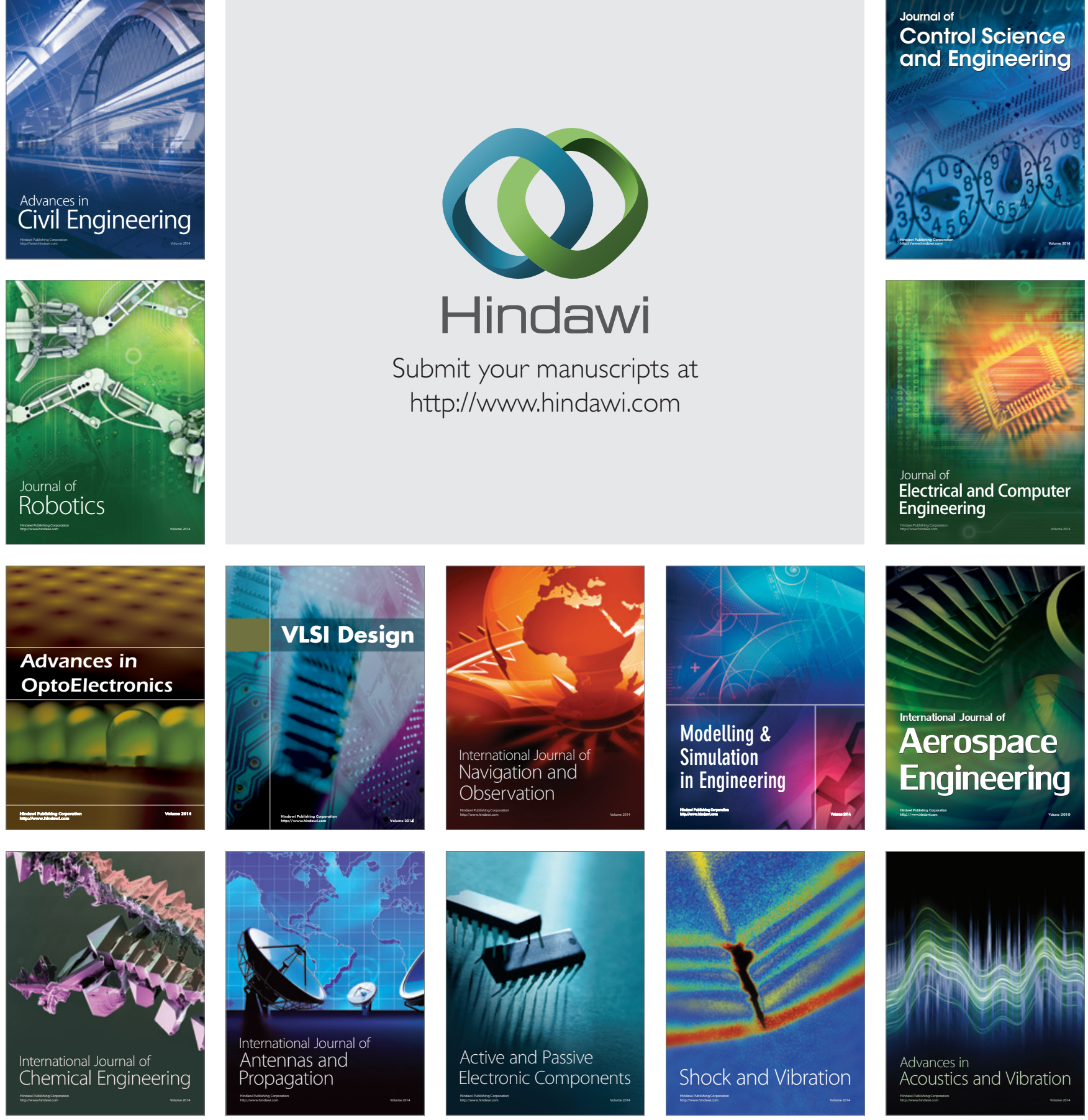